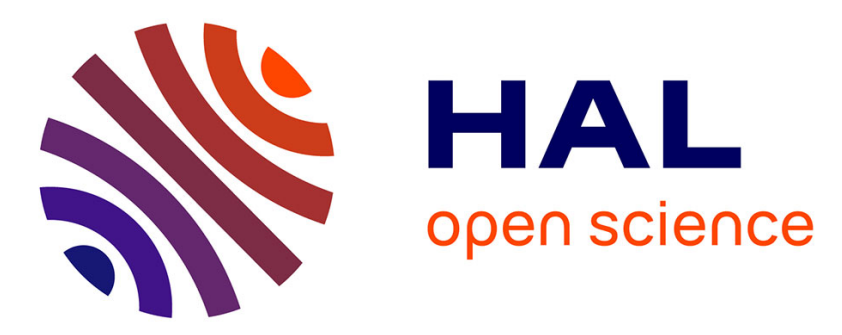

\title{
Insights into cation exchange selectivity of a natural clinoptilolite by means of dielectric relaxation spectroscopy
}

Gerardo Rodriguez-Fuentees, Sabine Devautour-Vinot, Sekou Diaby, François Henn

\section{To cite this version:}

Gerardo Rodriguez-Fuentees, Sabine Devautour-Vinot, Sekou Diaby, François Henn. Insights into cation exchange selectivity of a natural clinoptilolite by means of dielectric relaxation spectroscopy. Physics and Chemistry of Minerals, 2011, 38 (xx), pp.613. 10.1007/s00269-011-0433-4 . hal00607969

\section{HAL Id: hal-00607969 \\ https://hal.science/hal-00607969}

Submitted on 11 Jul 2011

HAL is a multi-disciplinary open access archive for the deposit and dissemination of scientific research documents, whether they are published or not. The documents may come from teaching and research institutions in France or abroad, or from public or private research centers.
L'archive ouverte pluridisciplinaire HAL, est destinée au dépôt et à la diffusion de documents scientifiques de niveau recherche, publiés ou non, émanant des établissements d'enseignement et de recherche français ou étrangers, des laboratoires publics ou privés. 


\title{
INSIGHTS INTO CATION EXCHANGE SELECTIVITY OF A NATURAL CLINOPTILOLITE BY MEANS OF DIELECTRIC RELAXATION SPECTROSCOPY
}

\author{
Gerardo Rodríguez-Fuentes $^{\mathrm{a}^{*}}$, Sabine Devautour-Vinot ${ }^{\mathrm{b}}$, Sekou Diaby ${ }^{\mathrm{c}}$ and François \\ Henn $^{\mathrm{b}}$
}

${ }^{a}$ Instituto de Ciencia y Tecnología de Materiales, Universidad de La Habana, Zapata y G s/n, Vedado, La Habana 10400, Cuba. gerardo@imre.oc.uh.cu

* corresponding author

${ }^{\mathrm{b}}$ Equipe Physicochimie des Matériaux Désordonnés et Poreux, Institut Charles Gerhardt, UMR 5253 CNRS, Université Montpellier 2, Place Eugène Bataillon, 34095 Montpellier cedex 5, France.devaut@1pmc.univ-montp2.fr

c Laboratoire de Chimie-Physique, UFR-SSMT (Unité de Formation et de Recherche en Sciences des Structures de la Matière et Technologie)

22 BP 582 Abidjan 22, Université de Cocody-Abidjan, Côte d'Ioire. sekoudiaby@ yahoo.fr

\begin{abstract}
.
Purified natural clinoptilolite from the Tasajeras deposit, Cuba, and some of its metal exchanged forms are studied, at the dehydrated state, by means of Dielectric Relaxation Spectroscopy (DRS) using two different modus operandi: Complex Impedance Spectroscopy and Dielectric Dynamic Thermal Analysis. Data analysis yields the determination of the extra-framework cation (EFC) population into the various possible crystallographic sites of the zeolitic framework as well as of the activation energy characterizing the localized hopping mechanism of EFC. First, it is shown that the DRS responses obtained here match well with the previous reported data which were previously localized EFCs in positions close to M1 and M2 sites when the clinoptilolite is modified to almost homoionic form. From this outcome, it can be concluded that all EFCs are in the same crystallographic situation regarding solvation or, in other terms, that no steric effect can be taken into account to explain cationic selectivity. Second, based on the assumption that the activation energy for EFC hopping is directly connected to the EFC/framework interaction and on simple thermodynamics consideration, we show this interaction does not govern the EFC
\end{abstract}


exchange reaction. So, it is emphasized that $\mathrm{EFC} / \mathrm{H}_{2} \mathrm{O}$ interaction is the key factor for cation exchange selectivity.

Keywords: natural clinoptilolite, dielectric relaxation, cation exchange 


\section{Introduction}

Cationic exchange is one of the most relevant properties of zeolites [1,2]. However, understanding of the microscopic mechanisms responsible for this property is still under debate and many questions remain posed. For instance, one may ask whether the localization of the extra-framework cations (EFC) at the inner-surface of the considered zeolites plays a key role into the cation selectivity. If so, it can be due either to i) steric effects, i.e. water molecules cannot solvate some given EFCs because of geometric restriction or the coordination sphere corresponding to the solvated cation is too large to cross some of the framework doorways, or to ii) energetic factors, i.e. depending on their position some EFC are too deeply trapped at the zeolites surface so that their solvation is not energetically favorable. In the later case, cationic exchange is therefore driven by the competition between $\mathrm{EFC} /$ framework and $\mathrm{EFC} /$ water interactions. In order to answer these questions, one needs to get deeper insights into EFC location at the surface and $\mathrm{EFC/framework} \mathrm{interaction.}$

Dielectric Relaxation Spectroscopy (DRS) was shown to be a powerful tool for investigating charge dynamics in ionic solids. In these solids, the dielectric response arises from the dipolar reorientation associated with the thermally activated ionic hops occurring between neighboring ionic positions. In zeolites, it has thus been well established that dielectric relaxation is associated with the localized displacement of EFC located at the surface in the microporosity of the crystalline structure [3]. It can then be expected that the dielectric relaxation properties of a given zeolite is strongly dependent on its framework features, i.e. crystallographic structure, $\mathrm{Si} / \mathrm{Al}$ ratio and localization of the $\mathrm{Al}$ atoms, and on the nature of EFC. DRS thus appears as a spectroscopy well adapted to characterize zeolites features along with XRD, Neutron diffraction, Infra-Red and NMR. More specially, it can be used to estimate the EFC localization among the various possible crystallographic ionic positions, the energy barrier associated to EFC hopping, later referred as the de-trapping energy, and, hence, the energy depth of the cationic sites.

Furthermore, it was shown that DRS is also very sensitive to the presence of guest molecules adsorbed in the nanoporosity where EFC sit [3-7]. This is particularly true when the adsorbed molecule is small and polar, e.g. water, and consequently when its interaction with EFC is significant. DRS is thus well suited to investigate water adsorption in zeolites. In that case, one of the key questions is to determine the precise role played by EFC in regards to other important parameters such as confinement in the zeolite nanoporosity and interaction of the adsorbed 
molecule with the non-cationic part of the inner-surface. The competitive interplay between the $\mathrm{EFC} / z e o l i t e s$ framework and the EFC/guest molecule interactions thus appears essential. This is particularly true when considering the zeolite properties and its Cationic Exchanged Capacity (CEC) in aqueous electrolytes. To that respect, DRS allows us to qualitatively compare the energy depth of the cationic sites that can be determined at the dry state with that of the cation solvation energy, and consequently to hierarchically distinguish the role played by the various interactions taking place upon hydration and ionic exchange. Besides, most of the dielectric studies carried out on zeolites have been conducted in synthetic types with high-symmetry. Only a few have been performed in natural ones as in heulandite-clinoptilolite [8], though this zeolite has lower symmetry and suffers from structural changes due to thermal effect and the evolution of the EFC locations. The dielectric response of a natural clinoptilolite from the Tasajeras deposit, Cuba, was studied by A. Delgado et al. [9]. They found that two dielectric relaxations take place at room temperature at about $34 \mathrm{~Hz}$ and $500 \mathrm{kHz}$ and that they could be associated to two different types of ionic hops.

In this paper, we aim at investigating the dielectric relaxation properties of a purified natural clinoptilolite $\mathrm{Nat}-\mathrm{Cli}$ and of its metal-exchanged forms, i.e. $\mathrm{Ca}^{2+}, \mathrm{Mg}^{2+}, \mathrm{K}^{+}$and $\mathrm{Na}^{+}$. DRS data are analyzed in terms of EFC de-trapping energy and of cationic population in the various crystallographic sites of the clinoptilolite framework. For that purpose, two DRS experimental procedures can be used: i) dielectric losses versus frequency under isothermal conditions and ii) dielectric losses at a given fixed frequency under thermal ramping. The later is called Dielectric Dynamic Thermal Analysis (DDTA). As already reported [4,10], the isothermal procedure allows us to determine the de-trapping energy, whereas the second one is more suitable for analyzing the shape of the dielectric signal. The de-trapping energy and the crystallographic EFC position are thus confronted to previous data obtained from structural investigations [22,23]. Finally, the cation de-trapping energy is compared both to that of the water solvation and to cationic selectivity.

The paper is constructed as followed. In section 2, the description of the samples studied here is given. A brief recall of DRS principle, data analysis and experimental procedure is provided in section 3. DRS results are discussed in section 4 where they are compared to the structural information provided by previous investigations, to the energy for cation solvation and to cationic selectivity. Conclusion is given in section 5 . 


\section{Description of the studied samples}

The natural zeolite from the Tasajeras deposit, Villa Clara province, Cuba, has been well characterized elsewhere [11]. This zeolite is a clinoptilolite, heulandite -IUPAC code HEU-. The exchanged forms of the purified natural clinoptilolite $\mathrm{Nat}-\mathrm{Cli}-\mathrm{Ca}^{2+}, \mathrm{Mg}^{2+}, \mathrm{K}^{+}$and $\mathrm{Na}^{+}$- are obtained by the hydrothermal exchange process using $1.0 \mathrm{~mol} . \mathrm{L}^{-1}$ aqueous solution of each metal. The zeolite powder is poured and then stirred in the electrolyte during 144 hours providing that the electrolyte was entirely replaced every 12 hours. The powder is washed with bi-distilled water until no trace of the electrolyte is detected. The cationic exchanged samples are noted: $\mathrm{Ca}-\mathrm{Cli}, \mathrm{Mg}-\mathrm{Cli}, \mathrm{K}-\mathrm{Cli}$ and $\mathrm{Na}-\mathrm{Cli}$. The chemical formula of each sample is determined by energy dispersive spectrometry using a Link System coupled to a JEOL 100CX electron microscope with ASID 4D scanning attachment. The crystallographic framework symmetry $\mathrm{C} 2 / \mathrm{m}$ is used for the calculation by fixing 72 oxygen atoms [12]. Chemical formulas thus obtained are reported in table 1.

\section{Brief recalls of DRS principle, data analysis and experimental procedure}

3.1 Basic principle and data analysis

Hereafter, DRS consists of measuring the sample response to an ac electrical field. The basic principles can be found in text books [13].

When a dipole relaxes because of local atomic re-arrangements, i.e. localized ionic hop in our case, the relaxation time associated to this movement is:

$\tau=\tau_{0} \exp \left[\frac{\Delta E}{k T}\right]$

where $k$ is the Boltzmann constant, $T$ the temperature, $\Delta E$ the activation barrier and $\tau_{0}$ the inverse characteristic vibration which is typical of atomic frequency, i.e. $10^{13} \mathrm{~s}^{-1}$.

Thus, following a first order relaxation mechanism, the dielectric loss spectrum $\varepsilon^{\prime \prime}(f, T)$ which characterizes the ac response measured at a given temperature $T$ and frequency $f$ is given by:

$\varepsilon^{\prime \prime}(f, T)=\frac{2 \pi f . \Delta \varepsilon}{1+(2 \pi f)^{2} \tau^{2}}$

where $\Delta \varepsilon$ is the so-called dielectric strength of the dipole. 
However, most of real systems are not characterized by a single type of dipole and consequently by a dielectric signal corresponding to eq.2. Instead, their dielectric response can be analyzed assuming there are different types of dipoles $i$ whose the respective populations can be described by a distribution function $G\left(\tau_{i}\right)$. Eq. 2 can thus be written:

$$
\varepsilon^{\prime \prime}(f, T)=\int_{0}^{\infty} G\left(\tau_{i}\right) \cdot \frac{2 \pi f \cdot \Delta \varepsilon_{i}}{1+(2 \pi f)^{2} \tau_{i}^{2}} \cdot d \tau
$$

For a population of dipoles which corresponds to ionic hops involving the same type of ions and of framework, $\tau_{0, i}$ and $\Delta \varepsilon_{i}$ can be assumed to be constant and equal to $\bar{\tau}_{0}$ and $\bar{\Delta} \varepsilon$ respectively. $\bar{\Delta} \varepsilon$ can then be factorized and $G\left(\tau_{i}\right)$ replaced, according to eq.1, by a distribution of activation energies $G\left(\Delta E_{i}\right)$ :

$$
\mathcal{E}^{\prime \prime}(f, T)=\bar{\Delta} \varepsilon \cdot \int_{0}^{\infty} G\left(\Delta E_{i}\right) \cdot \frac{2 \pi f}{1+(2 \pi f)^{2}\left[\bar{\tau}_{0} \cdot \exp \left(-\Delta E_{i} / k T\right)\right]^{2}} \cdot d \tau
$$

$G\left(\Delta E_{i}\right)$ characterizes the system under study and, in the present case, can be related to the various crystallographic sites in which EFC are located $[4,10,14]$ and around which they can hop. The fit of $\varepsilon^{\prime \prime}(f, T)$ with eq. 4 is an inverse and ill-posed problem $[15,16]$ so that no unique solution for $G\left(\Delta E_{i}\right)$ can be extracted. However, a "guessed" $G\left(\Delta E_{i}\right)$ function can be input into the minimization procedure based on some assumptions derived from the knowledge of the crystallographic structure of the studied zeolites. The minimization allows us to check whether the initial $G\left(\Delta E_{i}\right)$ fits well with the dielectric signal and, if so, to refine it. When $G\left(\Delta E_{i}\right)$ is refined, it gives us access to the mean activation energy and the relative population characterizing each different types of ionic hop. This procedure was proved to be productive in many zeolites $[4,10,14]$.

\subsection{Experimental procedure}

The zeolite powder is pressed to form disk-shape pellets whose diameter and area are $1 \mathrm{~mm}$ and $133 \mathrm{~mm}^{2}$ respectively. The zeolite pellet is sandwiched between two insulating layers [17], i.e. PTFE $10 \mu \mathrm{m}$ thin film, and placed between the metallic plates of the sample holder which is 
maintained under a continuous dry $\mathrm{N}_{2}$ flow. Prior to DRS measurements, the pellet is treated insitu at $220^{\circ} \mathrm{C}$ for 18 hours. So, it can be assumed that the studied sample remains totally dehydrated during the course of the experiment.

Ac permittivity is measured with a broadband dielectric spectrometer (Novocontrol BDS 4000), by using two experimental procedures (nb: the combination of two experimental procedures reduces the number of variables in the fitting procedure and, hence, makes it more reliable). In the first case, named Complex Impedance Spectroscopy (CIS) hereafter, the imaginary part of the permittivity, $\varepsilon_{T}^{\prime \prime}(f)$, is measured, under constant temperature ranging from $220^{\circ} \mathrm{C}$ to $70^{\circ} \mathrm{C}$, as a function of the ac electric field frequency in the $10^{-2} \mathrm{~Hz}-10^{6} \mathrm{~Hz}$ domain. In the second case, called Dielectric Dynamic Thermal Analysis (DDTA), the temperature evolution of the dielectric loss, $\varepsilon_{f}^{\prime \prime}(T)$ is measured at a fixed frequency $f$, while the temperature is ramping at a constant heating rate, i.e. $3^{\circ} \mathrm{C} \cdot \mathrm{min}^{-1}$, from $-50^{\circ} \mathrm{C}$ to $220^{\circ} \mathrm{C}$. The fixed frequency is chosen, accordingly to the sample response, so that the $\varepsilon_{f}^{\prime \prime}(T)$ peak is entirely scanned in the explored temperature domain. In the present study, $f$ turns out to be around 0.1 to $10 \mathrm{~Hz}$.

\section{Results and discussion}

\subsection{Analysis of the samples CIS and DDTA response}

Figure 1a reports a typical example of CIS signal, obtained in the case of $\mathrm{Na}-\mathrm{Cli} . \varepsilon_{T}^{\prime \prime}(f)$ exhibits a rather broad peak, accompanied at low frequency by a signal increase. The later corresponds to the so-called Maxwell Wagner Sillars effect which results from the accumulation of ionic charges at the sample/insulator layer interface and, hence, does not correspond to an intrinsic dielectric relaxation phenomenon. As expected from eq. 4, the dielectric relaxation peak shifts towards higher frequency upon increasing temperature. The same trend is observed for all the clinoptilolite samples studied in the present work.

The $\varepsilon_{T}^{\prime \prime}(f)$ peak maximum corresponds to $\tau_{\max }=\left(2 \pi f_{\max }\right)^{-1}$. The Arrhenius plot of $\tau_{\max }$, i.e. $\operatorname{Ln}\left(\tau_{\max }\right) \mathrm{vs} 1 / T$ (see figure $1 \mathrm{~b}$ ), can then be used to determine $\widetilde{\Delta} E$ (eq.1) the activation energy of the most probable EFC hop (figure 1b). Table 2 lists the values of $\tilde{\Delta} E$ for our different samples. The following classification can thus be made:

$$
\tilde{\Delta} E(K-C l i)=\tilde{\Delta} E(M g-C l i)>\widetilde{\Delta} E(N a t-C l i)=\tilde{\Delta} E(C a-C l i)>\tilde{\Delta} E(N a-C l i)
$$


It means that $\mathrm{Na}^{+}$cations are less deeply trapped at the surface of the dehydrated clinoptilolite framework than $K^{+}$ones are, or in other words that the interaction energy between the zeolite framework and the cation is lower for $\mathrm{Na}^{+}$than for $\mathrm{K}^{+}$. It can be also underlined that there is no direct correlation between the cation electric charge and the value of $\tilde{\Delta} E$.

It has already been pointed out that $\mathrm{K}-\mathrm{Cli}$ is more stable than $\mathrm{Na}-\mathrm{Cli}$ under high temperature treatment because $K^{+}$cations maintain the channel open upon dehydration, while $\mathrm{Na}^{+}$cations do not avoid the channel breakdown $[18,19]$. This observation is in accordance with the classification obtained from our DRS measurements (eq.5), which shows that $K^{+}$are deeper embedded than $\mathrm{Na}^{+} . \mathrm{K}^{+}$cations motion is therefore less responsive to temperature. Moreover, it has also been reported that the $\mathrm{Na}^{+}$-framework bond is weaker than that of the other EFC $[20]$.

$G\left(\Delta E_{i}\right)$ is determined from the fit of the DDTA signal, i.e. $\varepsilon_{f}^{\prime \prime}(T)$, which was shown to be more reliable than the fit of $\varepsilon_{T}^{\prime \prime}(f)$ obtained from the isothermal CIS method [4,10,21]. The value of $\Delta E_{\max }$, determined from CIS (see fig.1b), allows us to calculate the pre-exponential $\tilde{\tau}_{0}$ value from the maximum of the $\varepsilon_{f}^{\prime \prime}(T)$ peak and then to initiate the fitting procedure of the whole signal.

The $\varepsilon_{f}^{\prime \prime}(T)$ signal obtained on the dehydrated $\mathrm{Ca}-\mathrm{Cli}$ is reported in Figure 2. The increase of $\varepsilon_{f}^{\prime \prime}(T)$ at high temperature also corresponds to the so-called Maxwell-Wagner-Sillar effect as already mentioned in the case of $\varepsilon_{T}^{\prime \prime}(f)$ (fig. 1a). It is not an intrinsic feature of the sample and, hence, must not be taken into account in the experimental data analysis. First, we considered $G\left(\Delta E_{i}\right)$ as a simple Gaussian function whose the maximum value corresponds to the value of $\tilde{\Delta} E$ obtained from CIS. As the output was fairly poor, the addition of one more Gaussian function into $G\left(\Delta E_{i}\right)$ appeared unavoidable. Figure 3 illustrates $G\left(\Delta E_{i}\right)$ obtained for all samples. The shape and position of $G(\Delta E)_{i}$ differ according to the considered cation exchanged clinoptilolite sample. $G\left(\Delta E_{i}\right)$ characterizing $N a t-C l i$ is very similar in shape and position to that of $\mathrm{Ca}-\mathrm{Cli}$. This result is consistent with the fact that among the clinoptilolites 
studied here, the chemical composition of $\mathrm{Ca}-\mathrm{Cli}$ is the closest to that of the natural sample Nat - Cli (table 1)

In all cases, two Gaussian functions are necessary to recover the experimental data. This means that, independently on EFC, DDTA signal analysis reveals the presence of two distinct dipole populations. Table 3 compiles the parameters of $G\left(\Delta E_{i}\right)$ obtained from the fitting of each zeolite DDTA signal.

As described in figure 4, the possible cationic sites in a non-dehydrated natural clinoptilolite are noted M1, M2, M3 and M4. The M1, M3 and M4 sites are located in the 10 members ring cavity, while M2 is in the 8 members one. The occupation of these sites was experimentally determined from DRX by Koyama and Takeushi [22] and later by Alberti and Vessalini [23]. It was then concluded that $\mathrm{Na}^{+}$cations are located in $\mathrm{M} 1, \mathrm{Ca}^{2+}$ in $\mathrm{M} 2, \mathrm{~K}^{+}$in $\mathrm{M} 1$ and $\mathrm{M} 3$ and $\mathrm{Mg}^{2+}$ in M4, each of these cations having different number of water molecules in their first coordination shell. Furthermore, it was shown [24] that dehydration induces a displacement of the cations from M1 and M4 to M3 sites which is closer to the framework wall, meaning that in the dehydrated Nat-Cli only the M2 and M3 sites are occupied. The existence of two distinct cation locations as determined from all the DDTA signals is therefore in agreement with these former structural investigations.

In the case of the exchanged samples, the existence of two Gaussians function is also consistent with previous experimental works [22,23] and with computational simulations carried out by A.R. Ruiz-Salvador et al. [20,25,26]. In every case, the most occupied sites are M1 and M2 in dehydrated samples, with more accuracy in sites close to these experimentally determined position.

The relative area of each Gaussian function yields the relative population of cationic site, $\mathrm{cf}$ equation $4[4,14,21]$. Providing the incertitude due both to the experimental signal and to the fitting modus operandi, it can be concluded (see table 3) that, independently on their nature, the cations are equally distributed in these two sites. This important outcome also implies that during the exchange procedure, M1 and M2 sites being occupied in the same proportion, all EFC can be solvated in the same way without geometric restriction. So, there must be no steric effect due to the size of the cation hydration shell. 
As reported for the dry $\mathrm{Na}-\mathrm{Cli}$, the coordination number of the sodium cation with the oxygen atoms of the zeolite framework is lower for M1 than for M2 [25], i.e. the de-trapping energy is lower for M1 than for M2 cations. Therefore, it can be assumed that the Gaussian function characterized by the highest activation energy corresponds to EFC embedded in M2 sites whereas the second one is related to M1 sites.

\subsection{Relationship between the exchange selectivity and the de-trapping energy}

Since the de-trapping energy measured from DRS is directly related to the EFC/framework interaction, a correlation between its value and the EFC selectivity of the clinoptilolite could be anticipated. Indeed, it could be thought, at first sights, that the stronger is the EFC/framework interaction, the more difficult the cation extraction and, hence, the higher its selectivity. However, this too simple prospect does not take into account the fact that EFC are exchanged via the aqueous solution and, consequently, that a succession of solvation/desolvation steps are also involved. So, if we put apart steric effects, i.e. the accessibility of a given cation site, ionic selectivity must result from the competition between the EFC/framework and $\mathrm{EFC} / \mathrm{H}_{2} \mathrm{O}$ interaction. The comparison of both can therefore be useful for a better understanding of the cationic selectivity.

As reported in the literature [12,27], the exchange selectivity of Clinoptilolite is as follows:

$$
\mathrm{K}^{+}>\mathrm{Na}^{+}>\mathrm{Ca}^{2+}>\mathrm{Mg}^{2+}
$$

Eq. 6 means that, in the natural clinoptilolite, it is more difficult to exchange $K^{+}$than $M g^{2+}$. For $K^{+}$the situation is unambiguous since only $K^{+}$are present in the K-Cli sample (see table 1). For the other exchanged clinoptilolites, it is not possible to achieve such a high degree of cationic exchange. Again, according to the chemical composition reported in table 1, we can note that $\mathrm{Ca}^{2+}$ and to a less extend $\mathrm{Mg}^{2+}$ are easily replaced by $\mathrm{Na}^{+}$, while $\mathrm{Ca}-\mathrm{Cli}$ and $\mathrm{Mg}-\mathrm{Cli}$ still contain $\mathrm{Na}^{+}$. All these data are in full agreement with eq.6.

The question is now to correlate eqs $5 \& 6$, i.e. to check whether there is a correlation between cationic selectivity and cation de-trapping energy measured at the dry state. $K^{+}$appears to be the most deeply trapped cation (eq.5) at the dehydrated state and the less exchangeable one in aqueous solution (eq.6). At first sights, this outcome seems consistent if we assume that it is energetically favorable to exchange an ion by another one having stronger EFC/framework 
interaction, i.e. higher de-trapping energy. However, considering the other cations, the comparison of eqs. $5 \& 6$ does not provide clear evidence that this simple assumption is valid.

Indeed, cation exchange also involves solvation energy so that it is likely to rely on the competition between $\mathrm{EFC} /$ framework and $\mathrm{EFC} / \mathrm{H}_{2} \mathrm{O}$ interactions. A comparison between these two types of interaction appears therefore necessary.

According to the Nat-Cli chemical formula (table 1), EFC which can be exchanged for the greater part are $\mathrm{Ca}^{2+}$ and $\mathrm{Na}^{+}$. Then, the EFC exchange in aqueous solution can be featured by the two following chemical reactions:

$$
\begin{array}{rlrl}
\left(\mathrm{Na}^{+}\right)_{n}-\mathrm{Cli}+\mathrm{Y}^{n+}\left(\mathrm{H}_{2} \mathrm{O}\right)_{6} & =\left(\mathrm{Y}^{n+}\right)-\mathrm{Cli}+n N a^{+}\left(\mathrm{H}_{2} \mathrm{O}\right)_{6} & \Delta H_{\operatorname{exch}\left(\mathrm{Na} / \mathrm{Y}^{n+}\right)} \\
\left(\mathrm{Ca}{ }^{2+}\right)-\mathrm{Cli}+\frac{2}{n} \mathrm{Y}^{n+}\left(\mathrm{H}_{2} \mathrm{O}\right)_{6}=\left(Y^{n+}\right)_{\frac{2}{n}}-\mathrm{Cli}+\mathrm{Ca}^{2+}\left(\mathrm{H}_{2} \mathrm{O}\right)_{6} & \Delta H_{\operatorname{exch}\left(\mathrm{Ca} / \mathrm{Y}^{n+}\right)}
\end{array}
$$

for the $\mathrm{Na}^{+}$and $\mathrm{Ca}^{2+}$ exchange, respectively. In eqs.7, $\Delta H_{\text {exch }}$ is the corresponding exchanged reaction enthalpy. $\mathrm{Y}^{n+}\left(\mathrm{H}_{2} \mathrm{O}\right)_{6}$ represents the solvated cation, with $\mathrm{Y}=\mathrm{Na}^{+}, \mathrm{K}^{+}, \mathrm{Mg}^{2+}$ or $\mathrm{Ca}^{2+}$ and the cation valence, i.e. $\mathrm{n}=1$ or 2 . To make it simple, we assume that, in solution, the solvated cation is hexa-coordinated to water molecules, whatever its nature.

Noteworthy, we refer here to $\Delta H_{\text {exch }}$ and not to the free enthalpy $\Delta G_{\text {exch }}$ since it can be assumed that the entropy variation, $\Delta S_{\text {exch }}$, related to the cation exchange reaction is about the same for all EFC studied in this work. Therefore the chemical equilibrium symbolized by eqs.7 can be investigated using only $\Delta H_{\text {exch }}$.

The reaction enthalpy $\Delta H_{\text {exch }}$ (eqs.7) can be writen:

$$
\begin{aligned}
& \Delta H_{\text {exch }\left(\mathrm{Na} / \mathrm{Y}^{n+}\right)}=H_{\left(\mathrm{Y}^{n+}\right)-\mathrm{Cli}}+n H_{\mathrm{Na}^{+}\left(\mathrm{H}_{2} \mathrm{O}\right)_{6}}-H_{\left(\mathrm{Na}^{+}\right)_{n}-\mathrm{Cli}}-H_{\mathrm{Y}^{n+}\left(\mathrm{H}_{2} \mathrm{O}\right)_{6}} \\
& \Delta H_{\operatorname{exch}\left(\mathrm{Ca} / \mathrm{Y}^{n+}\right)}=H_{\left(\mathrm{Y}^{n+}\right)_{\frac{2}{n}}-\mathrm{Cli}}+H_{\mathrm{Ca}^{2+}\left(\mathrm{H}_{2} \mathrm{O}\right)_{6}}-H_{\left(\mathrm{Ca}^{2+}\right)-\mathrm{Cli}}-\frac{2}{n} H_{\mathrm{Y}^{n+}\left(\mathrm{H}_{2} \mathrm{O}\right)_{6}}
\end{aligned}
$$

where $H_{Y^{n+}\left(H_{2} O_{6}\right.}$ and $H_{\left(Y^{n+}\right)-C l i}$ correspond to the hydration of $Y^{n+}$ and to the $Y^{n+} /$ Framework interaction, respectively. Finally, eqs. 8 can simply reformulated:

$$
\Delta H_{\text {exch }}=\Delta H_{1}+\Delta H_{2}
$$


where $\Delta H_{1}$ and $\Delta H_{2}$ represent the difference of EFC solvation enthalpy and EFC/framework interaction respectively between the $\mathrm{Na}^{+}$and $\mathrm{Ca}^{2+}$ native and the exchanged $\mathrm{Y}^{n+}$ EFC. Thus, combining eqs (8) and (9), $\Delta H_{1}$ and $\Delta H_{2}$ can be expressed for the $\mathrm{Na}^{+}$and $\mathrm{Ca}^{2+}$ exchange reaction:

$$
\begin{aligned}
& \Delta H_{1\left(\mathrm{Na} / \mathrm{Y}^{n+}\right)}=n H_{\mathrm{Na}^{+}\left(\mathrm{H}_{2} \mathrm{O}\right)_{6}}-H_{Y^{n+}\left(\mathrm{H}_{2} \mathrm{O}\right)_{6}} \text { and } \Delta H_{2\left(\mathrm{Na} / \mathrm{Y}^{n+}\right)}=H_{\left(\mathrm{Y}^{n+}\right)-\mathrm{Cli}}-H_{\left(\mathrm{Na}^{+}\right)_{n}-\mathrm{Cli}} \\
& \Delta H_{1\left(\mathrm{Ca} / \mathrm{Y}^{n+}\right)}=H_{\mathrm{Ca}^{2+}\left(\mathrm{H}_{2} \mathrm{O}\right)_{6}}-2 / n H_{\mathrm{Y}^{n+}\left(\mathrm{H}_{2} \mathrm{O}\right)_{6}} \text { and } \Delta H_{2\left(\mathrm{Ca} / \mathrm{Y}^{n+}\right)}=H_{\left(\mathrm{Y}^{n+}\right)-\mathrm{Cli}}-H_{\left(\mathrm{Ca}^{2+}\right)-\mathrm{Cli}}
\end{aligned}
$$

$\Delta H_{1}$ can be determined from computations reported by various authors [29]. However, $\Delta H_{2}$ cannot be directly evaluated from our DRS experiments and, as far as we know, has never been computed in the cases studied here.

The energy barrier measured from DRS, i.e. $\tilde{\Delta} E$ (table 2), is expected to increase with the strength of the EFC/Framework interaction $H_{\left(Y^{n+}\right)-C l i}$ [14]. In other words, the variation of the $H_{\left(Y^{n+}\right)-C l i}$ must follow the same trend as the variation of the corresponding $-\tilde{\Delta} E_{\left(Y^{n+}\right)}(n b:$ the minus sign means that the lower is $H_{\left(Y^{n+}\right)-C l i}$, the larger $\left.\tilde{\Delta} E_{\left(Y^{n+}\right)}\right)$.

Consequently, we can establish the following qualitative connection between $\tilde{\Delta} E$ and $\Delta H_{2}$ :

$$
\begin{aligned}
& \Delta H_{2(\mathrm{Na})} \propto-\left(\tilde{\Delta} E_{\left(Y^{n+}\right)}-\tilde{\Delta} E_{\left(\mathrm{Na} a^{+}\right)}\right)=-\Delta(\tilde{\Delta} E)_{\left(\mathrm{Na}^{+} / Y^{n+}\right)} \\
& \Delta H_{2(\mathrm{Ca})} \propto-\left(\tilde{\Delta} E_{\left(Y^{n+}\right)}-\tilde{\Delta} E_{\left(\mathrm{Ca}^{+}\right)}\right)=-\Delta(\tilde{\Delta} E)_{\left({ }_{\left(a^{+}\right.} / Y^{n+}\right)}
\end{aligned}
$$

Table 4 resumes the values of $\Delta H_{1}$ and of $-\Delta(\tilde{\Delta} E)$ for all the possible cationic exchange reactions.

$\Delta H_{1}$ reported here are extracted from ref. 28 where they were calculated using the DFT (B3LYP/6-311++G(3d,3p)) method. Noteworthy, other values of $\Delta H_{1}$ can be found in the literature [see Tables I and II of ref 28] though the qualitative trend remains the same.

From eqs.10, it can be concluded that the higher is $\Delta H_{1}$, the more favorable the hydration of $\mathrm{Y}^{n+}$ with respect to $\mathrm{Na}^{+}$and $\mathrm{Ca}^{2+}$, meaning that the substitution of the later with $\mathrm{Y}^{n+}$ is not encouraged. Likewise, eqs.11 indicates that the higher is $-\Delta(\tilde{\Delta} E)$ the less favorable the trapping 
of $Y^{n+}$ at the surface of the zeolite framework. So, EFC corresponding to higher values of $\Delta H_{1}$ and of $-\Delta(\tilde{\Delta} E)$ are expected to be the less capable to substitute the native EFC.

We can now attempt to establish a qualitative comparison with the cation selectivity. First of all, we observe that in both cases, i.e. $\mathrm{Na}^{+}$and $\mathrm{Ca}^{2+}$ exchange reaction, the lowest value of $\Delta H_{1}$ is obtained for $\mathrm{K}^{+}$, meaning that $\mathrm{K}^{+}$hydration is not favored at all compared to $\mathrm{Na}^{+}$or $\mathrm{Ca}^{2+}$. Likewise, we note that $-\Delta(\tilde{\Delta} E)$ is lower for $K^{+}$, i.e. $K^{+}$is deeper trapped at the clinoptilolite surface. Therefore both energy contributions point out that $\mathrm{Na}^{+}$or $\mathrm{Ca}^{2+}$ can be easily exchanged by $\mathrm{K}^{+}$. Secondly, we can see that $\mathrm{Mg}^{2+}$ hydration is always the most preferential one whilst its corresponding values of $-\Delta(\tilde{\Delta} E)$ is equivalent to that of $K^{+}$. Thus, taking into account the chemical formula of $\mathrm{Mg}-\mathrm{Cli}$ and, to a less extent, that of $\mathrm{Na}-\mathrm{Cli}$, $\mathrm{K}-\mathrm{Cli}$ and $\mathrm{Ca}-\mathrm{Cli}$ which show that $\mathrm{Mg}^{2+}$ is the EFC that is the less easily trapped at the clinoptilolite surface, it can be emphasized that the main factor for $\mathrm{Mg}^{2+}$ exchange selectivity is the hydration enthalpy and not the EFC/Framework interaction. Finally considering the competition between $\mathrm{Na}^{+}$or $\mathrm{Ca}^{2+}$ cations, we can also observe that the exchange is mainly governed by the cation hydration enthalpy and not by the EFC/framework interaction. Indeed, though $\mathrm{Ca}^{2+}$ is more deeply trapped at the surface than $\mathrm{Na}^{+}$at the dehydrated clinoptilolite surface, i.e. $-\Delta(\tilde{\Delta} E)=-20 \mathrm{~kJ} . m o l-1$, the $\mathrm{Ca}^{2+} / \mathrm{H}_{2} \mathrm{O}$ interaction is so high compared to that of $\mathrm{Na}^{+}$, i.e. $\Delta H_{1}=141 \mathrm{~kJ} . \mathrm{mol}-1$, that all $\mathrm{Ca}^{2+}$ are replaced by $\mathrm{Na}^{+}$, the opposite being not true. It can be then concluded that, in all the cases investigated here, the cation exchange reaction enthalpy (eqs.7) is mainly governed by $\Delta H_{\text {exch }}$, the difference of hydration enthalpy. The differences in de-trapping energy between the various EFC examined in this work are not sufficient to drive the EFC exchange selectivity. To sum up, it means that, in our case, the EFC/framework interaction given by eq.5 cannot be compared to the cation selectivity featured by eq.6.

\section{Conclusion}

A series of metal exchanged clinoptilolites is studied by means of Dielectric Relaxation 
Spectroscopy (DRS). The signal analysis is shown to be in accordance with previous structural studies which emphasized the existence of two distinct types of extra-framework cation (EFC) position, i.e. close to M1 and M2 crystallographic sites, at the clinoptilolite inner-surface. Furthermore, analysis of the DRS signals makes possible the determination of the activation energy associated to the localized re-orientational motion of EFC. This activation energy is proportional to the ionic trapping energy and, hence, features the EFC/framework interaction. Cationic exchange capacity and cation selectivity properties of zeolites can be represented by a pseudo-chemical reaction (eq.7). It can then been assumed that, apart from specific steric effects, this chemical equilibrium is governed by the competition between the EFC/framework and $\mathrm{EFC} / \mathrm{H}_{2} \mathrm{O}$ interactions. The former is extracted from DRS analysis while the later can be determined from first principle computations already reported in the literature. Then, according to the experimental data reported on the cationic selectivity and to the chemical composition obtained for our sample after various cationic exchange (section 2), it turns out that $\mathrm{EFC} / \mathrm{H}_{2} \mathrm{O}$ interaction is the predominant parameter for cationic exchange in the clinoptilolite zeolites investigated in this work.

\section{Acknowledgements}

The authors thank the Universidad de La Habana for the support to this study. 


\section{References}

1. $\quad$ R.M. Barrer, J.A. Davies and L.V.C. Rees, J. Inorg. Nucl. Chem., 31 (1969) 2599.

2. $\quad$ R.M. Barrer and J. Klinowski, J. Chem. Soc. Faraday Trans. I, 68 (1972) 1956.

3. J.M. Kalogeras, A. Vassilikou-Dova, Cryst. Res. Technol. 1996, 31, 693.

4. A. Nicolas, S. Devautour-Vinot, G. Maurin, J.C. Giuntini \& F. Henn, J. Phys. Chem. C, 111(12), 4722 (2007).

5. J.C. Carru, D. Delafosse and M. Kermarec; J. Chim. Phys., Vo1.86. No.2. (1989) 263.

6. $\quad$ A. Szás, Al. Rahim and J. Liszi. Acta Chim. Hung., Vol. 125. No. 1 (1988) 37.

7. A.R. Haidar and A.K. Jonscher. J. Chem. Soc. Faraday Trans. 1. Vo1.82. No.12 (1986) 3535.

8. G. Rodríguez-Fuentes, A.R. Ruiz Salvador, M. Mir, O. Picazo, G. Quintana, M. Delgado, Mic. Mes. Mat. 20 (1998) 269.

9. A. Delgado, G. Rodríguez-Fuentes, A.R. Ruiz-Salvador, A. Berazain-Iturralde, IEEE Int. Symp. Elec, Ins. (1996) 866.

10. S. Devautour-Vinot, J.C. Giuntini, F. Henn, IEEE Transaction on Dielectrics and Electric Insulators 11, 320 (2004).

11. G. Rodríguez-Fuentes, L.C. de Ménorval, E. Reguera, F. Chávez, Microporous Mesoporous Materials 111 (2008) 577

12. G. Rodríguez-Fuentes, $\mathrm{PhD}$ thesis, Centro Nacional Investigaciones Científicas, Cuba (1987)

13. «Impedance Spectroscopy: Theory, Experiment, and Applications", E. Barsoukov and J.R. MacDonald Ed., J Wiley and son, 2005.

14. S. Devautour, J. Vanderschueren, J.C. Giuntini, F.Henn, J.V.Zanchetta \& J.L. Ginoux, J. Phys. Chem., B102, 3749 (1998)

15. H. Schäfer, E. Sternin, R. Stannarius, M. Arndt, F. Kremer, Phys. Rev. Lett., 76/12, (1996), 2177.

16. H. Schäfer, E. Sternin, La Physique au Canada, March-April, (1997), 77.

17. F. Henn, S. Devautour, L. Maati, J.C. Giuntini, J.V.Zanchetta, H.Schafer \& J.

Vanderschueren, Solid State Ionics 136-137, 1335 (2000)

18. T. Armbruster, M.E. Gunter Am Mineral 76 (1991) 1872.

19. M. Johnson, D. O’Connor, P. Barnes, C. Catlow, S. Owens, G. Sankar, R. Bell, S.J. Teat, R. Stephenson, J. Phys. Chem. B, 107, (2003) 942.

20. A.R. Ruiz-Salvador, A. Gómez, D.W. Lewis, R.A. Catlow, L.M. Rodríguez-Albelo, L. Montero and G. Rodríguez-Fuentes, Phys. Chem. Chem. Phys. 2 (2000) 1803.

21. A. Nicolas, S. Devautour-Vinot, J.C. Giuntini, G. Maurin and F. Henn, Microporous Mesoporous Materials 109 (2008) 413.

22. K. Koyama y Y. Takeushi, Z. Krystallogr 145 (1977) 216.

23. A. Alberti y G. Vessalini, TMPM Tschermrks Min. Petr. Mitt. 31 (1983) 259.

24. T. Armbruster, M.E. Gunter Am Mineral 76 (1991) 1872.

25. A.R. Ruiz-Salvador, D.W. Lewis, J. Rubayo-Soneira, G. Rodríguez-Fuentes, L. René and R.A. Catlow, J. Phys. Chem. B 102 (1998) 8417.

26. A.R. Ruiz-Salvador, A. Gómez, D.W. Lewis, G. Rodríguez-Fuentes and L. Montero, Phys. Chem. Chem. Phys. 1 (1999) 1679.

27. R. Woods, M. Gunter, Am. Mineralogist, 86, (2001), 424. 
28. J. S. Rao, T.C. Dinadayalane, J. Leszczynski, G.N. Sastry, J. Phys. Chem. A, 112, (2008), 12944. 
Table 1. Chemical formula of the purified natural clinoptilolite and of its cationic exchanged forms.

$$
\begin{aligned}
& N a t-C l i: \mathrm{Na}_{2.47} \mathrm{Ca}_{2.11} \mathrm{~K}_{0.94} \mathrm{Mg}_{0.41} \mathrm{Fe}_{0.93}\left(\mathrm{Al}_{6.86} \mathrm{Si}_{32.65} \mathrm{O}_{72}\right) 22 \mathrm{H}_{2} \mathrm{O} \\
& N a-C l i: \mathrm{Na}_{5.71} \mathrm{~K}_{0.69} \mathrm{Mg}_{0.18} \mathrm{Fe}_{0.41}\left(\mathrm{Al}_{6.99} \mathrm{Si}_{33.18} \mathrm{O}_{72}\right) 25 \mathrm{H}_{2} \mathrm{O} \\
& C a-C l i: \mathrm{Na}_{0.22} \mathbf{C a}_{5.29} \mathrm{~K}_{0.78} \mathrm{Mg}_{0.14} \mathrm{Fe}_{0.51}\left(\mathrm{Al}_{6.94} \mathrm{Si}_{29.58} \mathrm{O}_{72}\right) 22 \mathrm{H}_{2} \mathrm{O} \\
& K-C l i: \mathbf{K}_{6.73} \mathrm{Fe}_{0.36}\left(\mathrm{Al}_{7.09} \mathrm{Si}_{33.66} \mathrm{O}_{72}\right) 23 \mathrm{H}_{2} \mathrm{O} \\
& M g-C l i: \mathrm{Na}_{0.11} \mathrm{Ca}_{0.99} \mathrm{~K}_{0.68} \mathrm{Mg}_{4.33} \mathrm{Fe}_{0.84}\left(\mathrm{Al}_{6.95} \mathrm{Si}_{29.19} \mathrm{O}_{72}\right) 26 \mathrm{H}_{2} \mathrm{O}
\end{aligned}
$$


Table 2. Activation energy of the hopping process obtained from the Arrhenius plot for all

\begin{tabular}{|l|c|c|c|c|c|}
\hline samples & $N a-C l i$ & $C a-C l i$ & $N a t-C l i$ & $K-C l i$ & $M g-C l i$ \\
\hline$\tilde{\Delta} E$ in $(\mathbf{e V}) \pm 0.02$ & 0.78 & 0.99 & 0.99 & 1.06 & 1.06 \\
$\tilde{\Delta} E$ in $\left(\mathbf{k J}^{\prime} \mathbf{m o l}^{-1}\right) \pm 2$ & 75 & 95 & 95 & 102 & 102 \\
\hline
\end{tabular}


Table 3. Parameters of $G\left(\Delta E_{i}\right)$ determined the fit of the experimental DDTA signal with eq.4, assuming that $G\left(\Delta E_{i}\right)$ is the summation of two Gaussian functions. $\Delta E$ is the energy value at the Gaussian maximum and "Occupancy" is the area relative to the global distribution function

$$
G\left(\Delta E_{i}\right)
$$

\begin{tabular}{|c|c|c|c|c|}
\hline & \multicolumn{2}{|c|}{$1^{\text {st }}$ Gaussian } & \multicolumn{2}{c|}{$2^{\text {nd }}$ Gaussian } \\
\hline Sample & $\Delta E \pm \mathbf{0 . 0 2}$ & Occupancy & $\Delta E \pm \mathbf{0 . 0 2}$ & Occupancy \\
{$[\mathbf{e V}]$} & {$[\%]$} & {$[\mathbf{e V}]$} & {$[\%]$} \\
\hline$N a t-C l i$ & 0.99 & 49 & 0.90 & 51 \\
\hline$N a-C l i$ & 0.78 & 53 & 0.70 & 47 \\
\hline$C a-C l i$ & 0.99 & 60 & 0.88 & 40 \\
\hline$M g-C l i$ & 1.06 & 53 & 0.93 & 47 \\
\hline$K-C l i$ & 1.06 & 46 & 0.93 & 54 \\
\hline
\end{tabular}


Table 4. $\Delta H_{1}$ and $\Delta H_{2}$ (eqs. $9 \& 10$ ) for the exchange reaction of $\mathrm{Na}^{+}$and $\mathrm{Ca}^{2+}$ by $\mathrm{Y}^{n+}$ ions, with $\mathrm{Y}=\mathrm{Na}^{+}, \mathrm{K}^{+}, \mathrm{Mg}^{2+}$ or $\mathrm{Ca}^{2+} . \Delta H_{1}$ are determined from ref 27 (see tables I and II in [28]). $\Delta H_{2}$ is calculated using eqs. 11 from the values of $\tilde{\Delta} E$ reported in table 1

\begin{tabular}{|c|c|c|}
\hline$Y^{n+}$ & $\left(\mathrm{Na}^{+}\right)_{n}-\mathrm{Cli}+\mathrm{Y}^{n+}\left(\mathrm{H}_{2} \mathrm{O}\right)_{6}$ & $=\left(Y^{n+}\right)-C l i+n N a^{+}\left(\mathrm{H}_{2} \mathrm{O}\right)_{6}$ \\
\hline $\begin{array}{c}\mathrm{K}^{+} \\
\mathrm{Ca}^{2+} \\
\mathrm{Mg}^{2+} \\
\end{array}$ & $\begin{array}{c}\Delta H_{1(\mathrm{Na})} \\
(\mathrm{kJ} / \mathrm{mol}) \\
+104 \\
+141 \\
+446 \\
\end{array}$ & $\begin{array}{c}-\Delta(\tilde{\Delta} E)_{\left(N a^{+} / Y^{n+}\right)} \\
(\mathrm{kJ} / \mathrm{mol}) \\
-27 \\
-20 \\
-27\end{array}$ \\
\hline & $\left(\mathrm{Ca}^{2+}\right)-\mathrm{Cli}+2 / n \mathrm{Y}^{n+}\left(\mathrm{H}_{2} \mathrm{O}\right)$ & $=\left(\mathrm{Y}^{n+}\right)_{2 / n}-\mathrm{Cli}+\mathrm{Ca}^{2+}\left(\mathrm{H}_{2} \mathrm{O}\right)_{6}$ \\
\hline $\begin{array}{c}\mathrm{Na}^{+} \\
\mathrm{K}^{+} \\
\mathrm{Mg}^{2+}\end{array}$ & $\begin{array}{c}\Delta H_{1(\mathrm{Na})} \\
(\mathrm{kJ} / \mathrm{mol}) \\
-141 \\
-341 \\
+304\end{array}$ & $\begin{array}{c}-\Delta(\tilde{\Delta} E)_{\left({ }_{\left(a^{2+} / Y^{n+}\right)}\right.} \\
(\mathrm{kJ} / \mathrm{mol}) \\
+20 \\
-7 \\
-7\end{array}$ \\
\hline
\end{tabular}

